\title{
Correlation of Serum Potassium Status and HEART Score in Acute Coronary Syndrome Patients
}

\author{
Verina Logito, Nida Suraya, Dewi Kartika Turbawaty \\ Departement of Clinical Pathology, Faculty of Medicine Universitas Padjadjaran/Dr. Hasan \\ Sadikin General Hospital, Bandung, Indonesia
}

\section{Abstract}

Background: Patients with acute coronary syndrome (ACS) often experience hypokalemia which increase the risk of ventricular arrhythmia. The HEART Score can identify the prognosis of ACS. This study aimed to examine the correlation between serum potassium and HEART Score on ACS.

Methods: This was a cross-sectional observational, correlative analytic study. The data were taken retrospectively using secondary data. Inclusion criteria were all ACS patients who had been examined for serum potassium parameters at the Emergency Room of Dr. Hasan Sadikin General Hospital Bandung in 2019. The HEART Score was calculated based on history (H), electrocardiogram (E), Age (A), risk factors (R), and troponin (T). Subjects were divided into low risk, intermediate risk, and high risk groups based on the HEART Score value.

Results: Fifty-two ACS subjects were mostly male with a mean \pm SD age of $59 \pm 9$ years. All subjects had moderate and high HEART scores, with $79 \%$ subjects having normokalemia. There was no correlation between HEART Score and serum potassium levels $(r=-0.083, p=0.279)$.

Conclusion: There is no relationship between serum potassium and the HEART Score that may need to determine the prognosis in ACS patients. Further study is imperative to explore serum potassium levels which might begin to decline 24 hours after the acute attack.

Keywords: ACS, HEART Score, prognosis, serum potassium

\section{Introduction}

Acute coronary syndrome (ACS) is a cardiac emergency with discomfort in the chest or other symptoms, as a result of myocardial ischemia. Acute coronary syndrome comprises of unstable angina pectoris, non- $Q$ wave myocardial infarction or myocardial infarction without ST-segment elevation (Non-ST elevation myocardial infarction/NSTEMI), and $Q$ wave myocardial infarction or myocardial infarction with ST-segment elevation (STelevation myocardial infarction/STEMI. ${ }^{1}$

From year to year, cases of heart disease show an increase as the first cause of death in the world. Based on the results of Basic Health Research (Riset Kesehatan Dasar, Riskesdas) of the Ministry of Health of the Republic of Indonesia in 2018, the prevalence of coronary heart disease in Indonesia has reached $1.5 \%$ or around 2 million inhabitants. The prevalence is increased at the age over 45 years in men and after menopause in women. ACS was ranked second among 50 inpatient death ratings at the Dr. Hasan Sadikin General Hospital in Bandung, based on a diagnosis in 2017., ${ }^{1,2}$

Patients with ACS develop hypokalemia, which can increase the risk of lethal ventricular arrhythmias., ${ }^{3,4}$ Hypokalemia can be considered as an acute-phase response to adrenergic activation, which stimulates sodium-potassium-ATPase bonds and encourages potassium into cells. ${ }^{5}$ Another potential mechanism of hypokalemia in ACS is reactive hyperinsulinemia in response to elevated serum glucose due to adrenergic activity. Insulin will stimulate potassium to enter the muscles and liver, so insulin reduces the serum potassium concentration. If hypokalemia in ACS is driven primarily by adrenergic mechanisms, then in patients with diabetes, there is sympathetic nerve

Correspondence: Verina Logito, Department of Clinical Pathology, Faculty of Medicine, Universitas Padjadjaran/Dr. Hasan Sadikin General Hospital, Jalan Pasteur No. 38, Bandung, Indonesia, E-mail: verinalogito@gmail.com 
dysfunction associated with autonomic neuropathy. Thus, the potassium levels in ACS patients with diabetes will increase. ${ }^{6,7}$

Low-risk ACS patients can be well-managed in an outpatient clinic. Meanwhile, ACS patients with intermediate to high risk should be managed more quickly and aggressively, with hospitalization and consultation with a cardiology consultant. HEART Score aims to identify the risk of patient with undifferentiated chest pain that is useful for supporting medical decision-making by doctors. $^{8}$ The HEART Score is assessed based on five different variables, the scores being summed up for the patient being evaluated. These variables are history $(\mathrm{H})$, 12-lead electrocardiogram (E), age (A), risk factor (R), and troponin (T). The scores range from 0 to 2 in each of these five categories, with the lowest score of 0 and the highest score of 10 . The HEART score is divided into low risks (scores $0-3$ ), intermediate risks (scores 4-6), and high risks (scores 7-10). Therefore, the HEART Score can be used to determine prognosis in ACS patients. ${ }^{1,9}$ This study aimed to examine the relationship between serum potassium status and the HEART Score approach in acute coronary syndrome patients who were admitted to the Emergency Room of Dr. Hasan Sadikin General Hospital Bandung in 2019.

\section{Methods}

This research method was a cross-sectional study, using observational correlative analytic design. The total sampling method was employed. Retrospective data collection using secondary data taken from the Laboratory Information System (LIS) and patient medical records. The subjects of this study were patients with ACS who were admitted to the Emergency Room of Dr. Hasan Sadikin General Hospital Bandung in 2019. The inclusion criteria were unstable angina pectoris (UAP), ST-elevation myocardial infarction (STEMI) and non-ST-elevation myocardial infarction (NSTEMI) with and without risk factors. Data on serum potassium was collected. The exclusion criteria were patients with incomplete laboratory medical record data. The ethical clearance for this study was given by the Ethics Committee of Faculty of Medicine Universitas Padjadjaran/Dr. Hasan Sadikin General Hospital Bandung no LB.02.01/X.6.5/69/2020.

The data obtained were conducted with Spearman Rank Correlation Test to determine the relationship between serum potassium levels and the HEART Score approach in acute coronary syndrome patients, then the data were processed statistically with Microsoft Excel and SPSS 17.0.

\section{Results}

During 2019, 52 data of ACS patients who met the inclusion criteria were collected, consisting of 40 male and 12 female with a mean \pm SD age of $59 \pm 9$ years (range $37-83$ years). There were patients with UAP $(\mathrm{n}=12)$, NSTEMI $(\mathrm{n}=19)$, STEMI $(n=21)$.

The results of potassium levels were mostly $(77 \%)$ normal $(3.5-5 \mathrm{mg} / \mathrm{dl})$, and only 5 subjects were hypokalemia (2.9-3.4 mg/dl) and 6 other subjects with hyperkalemia (5.1$6.6 \mathrm{mg} / \mathrm{dl}$ ) as shown in Table 1.

The correlation of serum potassium with HEART Score using Spearman Rank analysis revealed a coefficient $r=-0.083 \quad(p=0.279)$, thus, there was a very weak and insignificant

Table 1 Characteristics of Patients with Acute Coronary Syndrome Admitted to Emergency Room, Dr. Hasan Sadikin General Hospital in 2019

\begin{tabular}{lc}
\hline \multicolumn{1}{c}{ Characteristics } & $\mathbf{n = 5 2}$ \\
\hline Age (year) & \\
$\quad$ Mean \pm SD & $59 \pm 9$ \\
$\quad$ Age criteria, n (\%) & \\
$\quad<45$ & $4(8)$ \\
$\quad 45-64$ & $36(69)$ \\
$\quad \geq 65$ & $12(23)$ \\
Gender, n (\%) & \\
Male & $40(77)$ \\
Female & $12(23)$ \\
Type of ACS, n (\%) & \\
Unstable angina & $12(23)$ \\
STEMI & $21(40)$ \\
NSTEMI & $19(37)$ \\
Potassium level, n (\%) & \\
Hypokalemia & $5(10)$ \\
Normokalemia & $41(79)$ \\
Hyperkalemia & $6(11)$ \\
HEART Score, n (\%) & \\
Low (0-3) & - \\
Moderate (4-6) & $16(31)$ \\
High (7-10) & $36(69)$ \\
\hline
\end{tabular}

Note: $\mathrm{SD}=$ standard deviation, $\mathrm{ACS}=$ acure coronsry syndrome, STEMI= ST-elevation myocardial infarction, NSTEMI = non-ST-elevation myocardial infarction 
Table 2 Proportions of Subjects with Moderate and High HEART Scores based on the Potassium Level

\begin{tabular}{ccccc}
\hline & Total & \multicolumn{2}{c}{ HEART Score } & P-value \\
\cline { 3 - 4 } & $\mathbf{n = 5 2}$ & $\begin{array}{c}\text { Moderate (4-6) } \\
\mathbf{n}(\%)\end{array}$ & $\begin{array}{c}\text { High (7-10) } \\
\mathbf{n}(\%)\end{array}$ & (\%) \\
\hline Potassium level & & & & $0.591^{*}$ \\
Hypokalemia & 5 & $1(2)$ & $4(8)$ & $27(52)$ \\
Normokalemia & 41 & $14(27)$ & $5(10)$ \\
Hyperkalemia & 6 & $1(2)$ & \\
\hline
\end{tabular}

Note: *Chi Square Test

negative correlation between serum potassium and HEART Score.

The proportion of subjects in the normokalemia group who had moderate HEART Score were 14 subjects (27\%) and high HEART Score were 27 subjects (52\%). There was no significant relationship between serum potassium and HEART Score ( $\mathrm{p}>0.591)$ (Table 2).

\section{Discussion}

Acute coronary syndrome (ACS) is the highest cause of death in the world. ${ }^{2}$ The incidence rate of ACS increases higher in women and men aged 55 to 64 years. ${ }^{10}$ This is in accordance with the characteristics of our respondents aged 59 years (range 37-83 years). Our study included 40 male and 12 female, supporting that male have a greater risk of suffering from ACS compared to female. The risk factor for ACS in male aged 45 years, while in female at age 55 years. ${ }^{11}$

Furthermore, most of the study subjects showed normal potassium levels, ranged from 3.5 to $4.9 \mathrm{mg} / \mathrm{dl}$. Score was $4.3 \mathrm{mg} / \mathrm{dl}$ (Range 3.4-6.5 mg/dl), and on a high HEART Score was $4.1 \mathrm{mg} / \mathrm{dl}$ (Range 2.9-6.6 mg/dl), and there was statistically no significant difference ( $>0.05$ ). Potassium levels $<3.5 \mathrm{mg} / \mathrm{dl}$ which has a moderate HEART Score of $2 \%$ and a high HEART Score of 8\%, at a potassium level of $3.5-5 \mathrm{mg} / \mathrm{dl}$ which has a moderate HEART Score of $27 \%$ and a high HEART Score of $52 \%$, at potassium levels $>5 \mathrm{mg} / \mathrm{dl}$ which had a moderate HEART Score of $2 \%$ and a high HEART Score of $10 \%$, statistically test showed no significant relationship ( $p>0.05$ ). Therefore, we can hypothesize that potassium levels are directly proportional to the HEART Score. In such manner, the higher the potassium level, the higher the HEART score produced.

Our study showed that the correlation coefficient between serum potassium and
HEART Score was -0.083 with $p=0.279(>0.05)$, meaning that there is no correlation between serum potassium and HEART Score.

Patients with ACS in acute attacks generally have transient hypokalemia, which can increase the risk of ventricular arrhythmias. ${ }^{12}$ Transient hypokalemia can be considered as an acutephase response to adrenergic activation, which stimulates sodium-potassium-ATPase bonds and pushes potassium into cells, resulting in most ACS patients with hypokalemia. ${ }^{12,13}$

Interestingly, other study shows there is a significant different in age for STEMI and NSTEMI group; as well as significant differences in sodium levels between STEMI and NSTEMI patients. ${ }^{14}$ However, the levels of potassium ( $\mathrm{p}=0.625)$ and chloride $(\mathrm{p}=0.423)$ did not have a significant difference between both groups. ${ }^{14}$

The results of this study, however, are not in line with the results of previous studies, resulting in the low percentage of hypokalemia caused by several factors, including low research subjects numbers, that dominated by normokalemia. ${ }^{12}$ The other factors are disruptive factor electrolyte levels, especially against potassium levels, which have not been considered before. Factors such as a history of diuretics and the presence of preexisting illnesses that affect potassium levels.

Imbalance in potassium levels does not take place quickly. In this study, the ACS patients were predominantly normokalemia $(n=41)$, and came to an acute attack, indicating that the imbalance in potassium levels does not take place immediately. Hypokalemia will occur 24 hours after the onset of ACS, when the patient is transferred to the Cardiac Intensive Care Unit (CICU) or when the doctor visit the CICU the next day. ${ }^{15,16}$ Therefore, further research is needed which measures potassium levels periodically.

The limitation of this study is that the periodic potassium checks are not conducted. 
Furthermore, there is no data on history of previous drug consumption and diseases that may affect potassium levels as a risk factor for the incidence of ACS.

In conclusion, there is a very weak and insignificant negative correlation between serum potassium and HEART Score to determine prognosis in ACS patients. There are many factors that influence the prognosis of ACS patients, among others potassium parameters. Therefore, it is recommended that further studies need to measure potassium regularly as well as to gather data on history of previous drugs and disease that may affect potassium level.

\section{References}

1. Nadeem M, Ahmed SS, Farooq S. Risk factors for coronary HEART disease in patients below 45 years of age. Pak J Med Sci. 2013;29(1):91-6.

2. World Health Organization. Cardiovascular disease (CVDs) [Internet] [cited 2021 May 6]. Available from: https://www. who.int/health-topics/cardiovasculardiseases\#tab=tab_1.

3. Xi H, Yu RH, Wang N, Chen XZ, Zhang WC, Hong T. Serum potassium levels and mortality of patients with acute myocardial infarction: a systematic review and metaanalysis of cohort studies. Eur J Prev Cardiol. 2019;26(2):145-56.

4. Ali M, Umer M, Butt UM, Tawwab S, Qureshi MA, Akram Z. Frequency of ventricular arrhythmias in acute myocardial infarction and its relationship with hypokalemia. J Cardiovasc Med Cardiol. 2018;5(4):036-8.

5. Iqbal R, Sami A, Ilyas M, Iftekhar MF, Ullah R, Arafat Y. Hypokalemia in patients with acute myocardial infarction. Pak Heart J. 2019;52(01):27-30.

6. Plakth Y, Gilutz H, Shiyovich A. The association of concomitant serum potassium and glucose levels and in-hospital mortality in patients with acute myocardial infarction (AMI). Soroka acute myocardial infarction II (SAMI-II) project. Int J Cardiol. 2019;287:39-45.

7. Faraj HR. Clinical study of some electrolytes (sodium, chloride and potassium) with patients in acute coronary syndrome (ACS) in ThiQar Governorate, Iraq. Int J Curr
Microbiol App Sci. 2015;4(3):700-5.

8. Six AJ, Backus BE, Kelder JC. Chest pain in the emergency room: value of the HEART Score. Neth Heart J. 2008;16(6):191-6.

9. Colombo MG, Kirchberger I, Amann U, Heier M, Thilo C, Kuch B, et al. Admission serum potassium concentration and long-term mortality in patients with acute myocardial infarction: results from the MONICA/ KORA myocardial infarction registry. BMC Cardiovasc Disord. 2017;17(1):198.

10. Mahmoud AN, Taduru SS, Mentias A, Mahtta D, Barakat AF, Saad M, et al. Trends of incidence, clinical presentation, and inhospital mortality among women with acute myocardial infarction with or without spontaneous coronary artery dissection: a population-based analysis. JACC Cardiovasc Interv. 2018;11(1):80-90.

11. Dong M, Mu N, Ren F, Sun X, Li F, Zhang C, et al. Prospective study of effect endogenous estrogens on myocardial no-reflow risk in postmenopausal women in acute myocardial infarction. J Interv Cardiol. 2014;27(5):437-43.

12. Sekiyama H, Nagoshi T, Komukai K, Matsushima M, Katoh D, Ogawa K, et al. Transient decrease in serum potassium level during ischemic attack of acute coronary syndrome: paradoxical contribution of plasma glucose level and glycohemoglobin. Cardiovasc Diabetol. 2013;12:4.

13. Barkas F, Elisaf M. Serum potassium levels and mortality in acute myocardial infarction: myth or fact? Angiology. 2018;69(8):657-9.

14. Ciptono F, Rahayu M. The differences of sodium, potassium and chloride levels in STEMI and NSTEMI patients. Indones J Clinical Pathol Med Laboratory. 2017;24(1):91-4.

15. Madias JE, Shah B, Chintalapally G, Chalavarya G, Madias NE. Admission serum potassium in patients with acute myocardial infarction: its correlates and value as a determinant of in-hospital outcome. Chest. 2000;118(4):904-13.

16. Uluganyan M, Ekmekçi A, Murat A, Avşar Ş, Ulutaş TK, Uyarel $H$, et al. Admission serum potassium level is associated with in-hospital and long-term mortality in STelevation myocardial infarction. Anatol J Cardiol. 2016;16(1):10-5. 